\title{
SUBJECT INDEX 19th RADIOCARBON CONFERENCE PROCEEDINGS, VOL 49(2), 2007
}

$\delta^{13} \mathrm{C}, 767-773$

${ }^{14} \mathrm{C}$ and ${ }^{13} \mathrm{C}, 807-816$

${ }^{14} \mathrm{C}$ calibration, $393-401,447-457,855-864$

${ }^{14} \mathrm{C}$ calibration curve, $1123-1132$

${ }^{14} \mathrm{C}$ concentration, $1045-1053$

${ }^{14} \mathrm{C}$ dating, 201-210, 211-216, 217-224, 225-232, 281-

289, 301-306, 307-314, 349-356, 499-514, 543-550, $617-623,625-637,645-658,673-684,703-712,757-$ 766, 799-806, 827-835, 947-961, 993-1001, $1003-$ 1015, 1023-1030, 1055-1063, 1065-1078, 1103-1121, $1145-1151$

${ }^{14} \mathrm{C}$ dating close to background, $379-391$

${ }^{14} \mathrm{C}$ half-life, $441-445$

$\Delta^{14} \mathrm{C}, 767-773$

2nd millennium BCE, 481-497

Absolute dating in archaeology, 465-472

Accelerator mass spectrometry [AMS], 225-232, 233244, 245-254, 255-269, 301-306, 349-356, 369-377, $441-445,459-464,473-479,551-564,727-740,789$ 798

$\alpha$-cellulose, 379-391

Adobe, 551-564

Agathis Australis, 447-457

Age anomaly, 817-826

Agricultural terraces, 481-497

Aqueduct, 481-497

Archaeology, 543-550

Archaeomagnetic dating, 543-550

Atmosphere, 807-816, 1031-1043

Atmospheric exchange, 969-982

Atmospheric nuclear explosions, 905-914

Atmospheric ${ }^{14} \mathrm{C}$ variation, 473-479, 1031-1043

Automated sample preparation, 233-244

Background optimization, 271-280, 315-323

Baelo Claudio, 827-835

Baja California, 899-904

Balochistan, 703-712

Baltic Sea, 527-542

Bayesian age modeling, 357-367, 369-377, 565-578

BEAGLE2003, 937-945

Benzene polycarboxylic acids, 1079-1091

Biofuel, 325-330

Black carbon, 1079-1091

Blank correction, 379-391

Blue optically stimulated luminescence (B-OSL), 11451151

Bomb ${ }^{14} \mathrm{C}, 937-945,1055-1063$

Bone collagen, 187-192, 193-200, 201-210, 579-592

Bronze Age, 713-726

Bulk sediment, 983-992

Burials, 727-740

Cabo de Gata, 827-835
Carbon accumulation, 1055-1063

Carbon cycle, 1055-1063

Carbon dioxide, 349-356, 807-816, 993-1001

Carbonate dissolution, 969-982

Cedar, 1045-1053

Chanca, 579-592

Charcoal samples, 611-616, 659-672

Chauvet Cave, 339-347

Chemical pretreatment, $855-864$

Chronology, 551-564, 565-578, 625-637, 645-658, 703-712, 757-766

Climate, 741-756, 775-788, 837-854, 889-897, 11031121

$\mathrm{CO}_{2}$ collection, 233-244

Coastal upwelling, 925-936

Compact AMS, 307-314

Consensus ages, $427-440$

Continuous autoregressive process, 369-377

Corals, 905-914

Core sampling, 1045-1053

Cosmogenic and stable isotopes, 827-835

Crannogs, 673-684

Cremated bone, 403-408, 499-514

Cultural dynamics, 1103-1121

Dajiuhu Basin, 789-798

Danube Basin, 727-740

Data analysis, 369-377, 393-401

Dendrochronology, 331-337, 617-623, 693-702

Direct absorption method, 281-289

Dissolved organic matter, 767-773

Dunes, 1145-1151

Earthquakes, 827-835

East Asian monsoon, 865-875

Eastern Central China, 789-798

Elite burial, 685-691

Environmental changes, 789-798, 799-806

Evasion, 993-1001

Flooding sediments, 611-616

Flue gas, 325-330

Fortresses, 481-497

Fossil fuel, 325-330, 807-816

Frequency distributions, 799-806

Freshwater reservoir effect, 947-961

FTIR, 201-210, 211-216

Galicia, 925-936

Gas handling system, 307-314

Gas ion source, 307-314

Golan Heights, 625-637

Graphite sample preparation, 217-224, 245-254, 255269

Graphitization, 245-254 
HBCO correction, 465-472

Hekla-3 tephra, 357-367

High-sensitivity ${ }^{14} \mathrm{C}$ dating, 379-391

Holocene, 527-542, 799-806, 899-904, 1017-1021

Holocene eruptions, 1065-1078

Holocene optimum, 865-875

Human bone collagen offset, 465-472

Human influences, 789-798

Human migrations, 527-542

Human remains, 593-610

Human society and civilizations, 827-835

Hungary, 515-526, 1017-1021, 1023-1030, 1031-1043

Hun-Sarmatian period, 693-702

Iceland, 659-672, 947-961

Intercomparison, 339-347, 403-408, 409-426, 427-440

Ion source, 301-306

Israel, 625-637, 1003-1015

Japan Sea, 915-924

Japan Sea bottom water, 915-924

Lacustrine environment, 767-773, 889-897, 983-992, 1017-1021, 1133-1143

Ladoga Lake, 527-542

Lake Wigry, 1133-1143

Larix sibirica, 693-702

Last deglacial, 963-968

Last Glacial age, 1123-1132

Late Glacial, 799-806

Late Holocene, 789-798

Liquid scintillation spectroscopy (LSC), 281-289, 315323, 379-391

Lithuania, 889-897

Lochs, 673-684

Loess, 1023-1030

London, 593-610

Marine environment, 817-826

Marine reservoir effect, 877-888, 899-904, 905-914, 925-936, 947-961, 963-968

Marine shells, 925-936

Marker tephra beds, 1065-1078

Measurements, 291-299

Medieval, 593-610, 639-644, 659-672

Melanopsis, 1003-1015

Mollusks, 877-888, 889-897

Mongolian Empire, 685-691

Monitoring, 281-289

Mostar, 617-623

Multiproxy analysis, 865-875

Mummies, 565-578

Nasca, 551-564

Negev, 481-497

Neolithic, 727-740

New Zealand, 447-457, 1093-1102
Nitrous oxide, 245-254

Norse, 659-672, 947-961

Northwestern Pacific, 963-968

Nuclear power plant, 1031-1043

Ocean circulations, 905-914, 915-924

Old Bridge, 617-623

Oracle bone, 211-216

Oxalic acid I (OX-I), 441-445

Oxygen combustion, 271-280

Oxygen isotope stage $3,447-457$

Paleodiet, 713-726

Paleolithic, 741-756, 757-766

Paracas, 551-564

Parietal art, 339-347

Pazyryk culture, 693-702

Peat deposits, 357-367, 789-798, 1055-1063

Perth, 639-644

Peru, 565-578, 579-592, 877-888

Physical anthropology, 465-472

Pine, 1045-1053

Plant fixation, 993-1001

Poland, 799-806, 1133-1143

Pottery, 639-644

Precision dating, 331-337

Prehistoric archaeology, 579-592

Pretreatment, 211-216

Proxy records, 827-835

Rare-earth elements, 1145-1151

Regional offset, 331-337, 473-479

Reliability, 427-440

Repeat hydrography, 937-945

Riparian, 993-1001

Russia, 693-702, 713-726

Sample preparation, 217-224, 225-232, 291-299

Sedimentation rate, 983-992

Shells, 1003-1015

Siberia, 645-658, 741-756, 757-766, 1103-1121

Sinai, 481-497

Skeletal age classes, $465-472$

Small $\mathrm{CO}_{2}$ samples, 307-314

Small organic samples, 271-280

Snails, 1145-1151

Soil carbon dynamics, 1079-1091, 1093-1102

Soil chronosequence, 1093-1102

Solar activity, 459-464, 827-835, 1123-1132

South China Sea, 905-914

South Kamchatka, 1065-1078

Spain, 543-550

St. Mary Spital, 593-610

Stable isotopes, 233-244, 645-658, 727-740

Statistics, 527-542

Stratigraphic sequences, 611-616

Stream profile, $969-982$ 
Submilligram samples, 255-269

Subtropical South Pacific, 937-945

Sue ware, 331-337

Suess effect, 775-788

Surface/depth ratio, 969-982

Swifterbant culture, 357-367

Tavan Tolgoi, 685-691

Terrestrial gastropods, $817-826$

Time-transgressive, 865-875

Tisza River, 515-526

Towada, 1123-1132

Tree rings, $459-464,473-479,775-788,855-864$,

1045-1053, 1123-1132

Tsunami, 827-835

Turks, 693-702

Two Creeks, 855-864
Typochronology, 499-514

Ultrafiltration, 187-192, 193-200

Uncertainty, 349-356, 427-440

Upper Paleolithic, 339-347

Upwelling, 877-888

Urnfields, 499-514

US Midwest, 855-864

Vegetation, 1103-1121

VIRI, 403-408, 409-426

Wari, 579-592

Water sample preparation, 281-289

Wetland, 983-992

Wiggle-match dating, 331-337, 357-367

WOCE-P06, 937-945 